\title{
The Deceptively Boring PSR J1738+0333
}

\author{
PAULO FREIRE* \& NORBERT WEX \\ Max-Planck-Institut für Radioastronomie \\ Auf dem Hügel 69, Bonn, 53121, Germany \\ *E-mail: pfreire@mpifr-bonn.mpg.de
}

\begin{abstract}
We present preliminary results of 7 years of Arecibo timing of the pulsar-white dwarf binary PSR J1738+0333. We can measure the proper motion, parallax with excellent precision and have detected the orbital decay. Furthermore, the companion has been detected at optical wavelengths and a mass ratio of $8.1 \pm 0.3$ has been measured from the orbital variation of its Doppler shift. Once the companion mass is determined from the optical measurements, this system will provide strong limits for the radiation of dipolar gravitational waves. Assuming that general relativity holds, the fast-improving measurement of the orbital decay, combined with the measurement of the mass ratio, will provide an independent and precise measurement of the component masses.
\end{abstract}

Keywords: PSR J11738+0333; dipole radiation; neutron star masses.

\section{Introduction}

PSR J1738+0333 is a $5.85 \mathrm{~ms}$ pulsar in a low eccentricity binary system with orbital period of $P_{b}=8.5 \mathrm{~h}^{1}$ and a low mass helium white dwarf (WD) companion. The pulsar was discovered in 2001 in a $20-\mathrm{cm}$ intermediate Galactic latitude survey ${ }^{11}$ carried out with the multi-beam system of the Parkes Telescope. Given its low Northern declination, it can be observed with much improved sensitivity using the the 305-m Arecibo Telescope. For that reason it has been timed with Arecibo since 2003 using the L-wide receiver (with frequency coverage from 1100 to 1700 $\mathrm{MHz}$ ). We used the Wide-band Arecibo Pulsar Processor (WAPP) correlators and the Arecibo Signal Processor (a coherent dedispersion machine) as back-ends. The WAPPs have bandwidths of $100 \mathrm{MHz}$ centered at 1170, 1410 and $1510 \mathrm{MHz}$ (and, occasionally, a fourth WAPP centered at $1310 \mathrm{MHz}$ as well).

The WAPP correlator data were Fourier transformed to obtain one 256-point power spectrum every $64 \mu \mathrm{s}$. These are then folded and dedispersed at the pulsar's spin period and DM using "sigfoldpow", a program designed by Ingrid Stairs specifically for producing highly accurate average pulse profiles from search data. The ASP data have not yet been fully analyzed, that is one of the reasons why these results are still preliminary. The observed pulse profiles are then cross-correlated with a low-noise template, the best fit yields a pulse time of arrival at the telescope (TOA). 
The TOAs were then analysed using the TEMPOa timing software, which makes a least-squared fit of the observed barycentric TOAs to a timing model, which includes spin, astrometric and orbital parameters.

\section{Results}

We have obtained a TOA residual rms of $1.4 \mu \mathrm{s}$, with TOAs taken every 268 $\mathrm{s}$ for each of the 12 (or 16) simultaneous $12.5-\mathrm{MHz}$ sub-bands. This results in more than 16000 TOAs. Of these, we use 9000 with a stated accuracy better than $3 \mu$ s. This large number of precise TOAs provide extraordinarily precise timing parameters. These include the proper motion $\left(\mu_{\alpha}=7.041 \pm 0.007\right.$ mas yr $^{-1}$

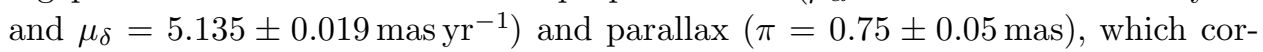
responds to a distance of $d=1.33 \pm 0.09 \mathrm{kpc}$. The orbital period is measured so precisely $\left(P_{b}=30653.9194513 \pm 0.0000005 \mathrm{~s}\right)$ that we can already detect the orbital decay, albeit to only $2-\sigma$ precision: $\dot{P}_{b}^{\text {obs }}=(-2.1 \pm 1.0) \times 10^{-14} \mathrm{~s} \mathrm{~s}^{-1}$. This becomes more significant after subtracting the extrinsic orbital period variation caused by the proper motion of the system ${ }^{2}$ and the Galactic acceleration: $\dot{P}_{b}^{\text {ext }}=(0.73 \pm 0.08) \times 10^{-14} \mathrm{~s} \mathrm{~s}^{-1}$. The intrinsic orbital period derivative is $\dot{P}_{b}^{\text {int }}=(-2.9 \pm 1.0) \times 10^{-14} \mathrm{~s} \mathrm{~s}^{-1}$.

\section{Optical Observations}

The companion white dwarf has been detected, and it has deep spectral lines typical of a $\sim 0.2 M_{\odot}$ thick-envelope He white dwarf. These were used to determine a radial-velocity curve using Gemini South on Cerro Pachón. From this we can derive the mass ratio of the system, $R=8.1 \pm 0.3$ (Van Kerkwijk, 2007, pers. comm.). At the moment we don't have a good estimate of the companion mass. However, if it follows the relation postulated by Tauris \& Savonije in $1999, \frac{3}{1}$ then the companion mass $m_{c} \simeq 0.18 \pm 0.02 M_{\odot}$, which is qualitatively consistent with the observed spectral properties. Given the mass ratio this results in a pulsar mass $m_{p} \simeq 1.46 \pm 0.16 M_{\odot}$ and an orbital inclination $i \simeq 33^{\circ}$. Apart from resulting in a reasonable value for $m_{p}$, the low inclination is consistent with the non-detection of the Shapiro delay. Furthermore, when we introduce Shapiro delay parameters $m_{c}$ and $\sin i$ in our solution, the apparent eccentricity diminishes to an insignificant level of $0.00000039 \pm 0.00000013$. This implies that the difference between the semi-major and semi-minor axes is only $8 \pm 6 \mu \mathrm{m}$ !

\section{Limit on dipolar gravitational wave emission}

If these values for $m_{c}$ and $m_{p}$ hold, the orbital decay due to the emission of quadrupolar gravitational waves predicted by general relativity is $\dot{P}_{b}^{\text {GR }}=-2.7 \times$ $10^{-14} \mathrm{~s} \mathrm{~s}^{-1}$. This is consistent with the intrinsic orbital decay $\dot{P}_{b}{ }^{\text {int }}$.

$\overline{\text { http://www.atnf.csiro.au/research/pulsar/tempo/ }}$ 


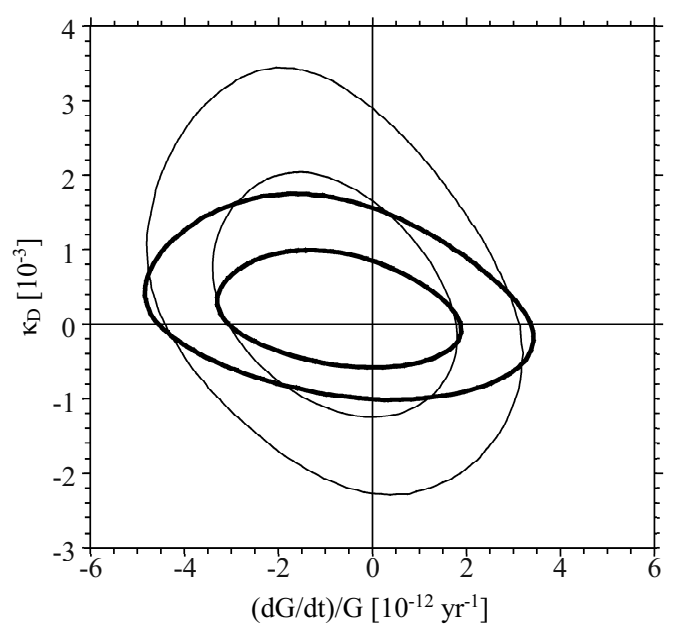

Fig. 1. Limits on $\dot{G}$ and $\kappa_{D}$ derived from the joint analysis of the timing data for PSR J0437-4715 plus PSR J1012+5307 (thin contours) or PSR J1738+0333 (thick contours). The inner contours include $68.3 \%$ and the outer contours include $95.4 \%$ of the simulated probability in each case.

Subtracting $\dot{P}_{b}^{\text {GR }}$ from $\dot{P}_{b}^{\text {int }}$ we obtain an "excess" of $\dot{P}_{b}^{\text {exc }}=(-0.2 \pm 1.0) \times$ $10^{-14} \mathrm{~s} \mathrm{~s}^{-1}$. Following the logic of Lazaridis et al. (these proceedings), we can combine this "excess" with that from a pulsar with a longer orbital period, like PSR J0437-4715, $\frac{5}{5}$ and derive joint limits for the variation of the gravitational constant $\dot{G}$ and the dipolar gravitational wave emission constant $\kappa_{D}$. In figure 1, we can see that, provided that the companion mass estimate is realistic, PSR J1738+0333 already provides excellent limits on dipolar gravitational wave emission: $\kappa_{D}=1.4_{-4.7}^{+5.6} \times 10^{-4}(1-\sigma)$ and $\kappa_{D}=1.4_{-9.3}^{+13.3} \times 10^{-4}(2-\sigma)$. This happens despite it having a baseline that is only half that of PSR J1012+5307: this is more than compensated by its shorter orbital period and better timing precision. This also means that the measurement of $\dot{P}_{b}{ }^{\text {int }}$ will keep improving much faster for PSR J1738+0333 in the future. One of the consequences is that, if we assume general relativity to hold (i.e., $\dot{P}_{b}^{\text {int }}=\dot{P}_{b}^{\text {GR }}$ ), we will obtain precise values for $m_{c}$, $m_{p}$ and $i$ from the measurements of $\dot{P}_{b}^{\text {GR }}$ and $R$. These will help to calibrate the spectral models of low-mass WDs.

\section{References}

1. Jacoby, B. A., Bailes, M., Ord, S. M., Knight, H. S., \& Hotan, A. W. 2007, Ap.J., 656, 408

2. I. S. Shklovskii, Soviet Ast. 13, 562 (1970).

3. Tauris, T. M., \& Savonije, G. J. 1999, A $\& A$, 350, 928

4. K. Lazaridis, N. Wex, A. Jessner and et al., MNRAS 400, 805 (2009).

5. J. P. W. Verbiest, M. Bailes, W. van Straten and et al., ApJ 679, 675 (2008). 